\title{
Cooperative colorings of trees and of bipartite graphs
}

\author{
Ron Aharoni* \\ Department of Mathematics \\ Technion - Israel Institute of Technology \\ Technion City, Haifa 3200003, Israel \\ ra@tx.technion.ac.il \\ Maria Chudnovsky \\ Mathematics Department \\ Princeton University \\ Princeton, NJ 08544, USA \\ mchudnov@math.princeton.edu \\ Eli Berger ${ }^{\dagger}$ \\ Department of Mathematics \\ University of Haifa \\ Mt. Carmel, Haifa 3498838, Israel \\ berger@math.haifa.ac.il \\ Frédéric Havet \\ CNRS, Université Côte d'Azur, \\ I3S, and INRIA \\ Sophia-Antipolis Cedex 06902, France \\ frederic.havet@inria.fr \\ Zilin Jiang $\S$ \\ Department of Mathematics \\ Massachusetts Institute of Technology \\ Cambridge, MA 02139, USA \\ zilinj@mit.edu
}

Submitted: Aug 20, 2018; Accepted: Jan 20, 2020; Published: Feb 7, 2020

(C) The authors. Released under the CC BY-ND license (International 4.0).

\begin{abstract}
Given a system $\left(G_{1}, \ldots, G_{m}\right)$ of graphs on the same vertex set $V$, a cooperative coloring is a choice of vertex sets $I_{1}, \ldots, I_{m}$, such that $I_{j}$ is independent in $G_{j}$ and $\bigcup_{j=1}^{m} I_{j}=V$. For a class $\mathcal{G}$ of graphs, let $m_{\mathcal{G}}(d)$ be the minimal $m$ such that every $m$ graphs from $\mathcal{G}$ with maximum degree $d$ have a cooperative coloring. We prove that $\Omega(\log \log d) \leqslant m_{\mathcal{T}}(d) \leqslant O(\log d)$ and $\Omega(\log d) \leqslant m_{\mathcal{B}}(d) \leqslant O(d / \log d)$, where $\mathcal{T}$ is the class of trees and $\mathcal{B}$ is the class of bipartite graphs.
\end{abstract}

Mathematics Subject Classifications: 05C15, 05C69

*Supported in part by the United States-Israel Binational Science Foundation (BSF) grant no. 2006099, the Israel Science Foundation (ISF) grant no. 2023464 and the Discount Bank Chair at Technion. This paper is part of a project that has received funding from the European Union's Horizon 2020 research and innovation programme, under the Marie Skłodowska-Curie grant agreement no. 823748.

${ }^{\dagger}$ Supported in part by BSF grant no. 2006099 and ISF grant no. 2023464.

${ }^{\ddagger}$ Supported in part by BSF grant no. 2006099, NSF grant DMS-1550991 and US Army Research Office Grant W911NF-16-1-0404.

$\S$ The work was done when Z. Jiang was a postdoctoral fellow at Technion - Israel Institute of Technology, and was supported in part by ISF grant nos. 409/16, 936/16. 


\section{Introduction}

A set of vertices in a graph is called independent if no two vertices in it form an edge. A coloring of a graph $G$ is a covering of $V(G)$ by independent sets. Given a system $\left(G_{1}, \ldots, G_{m}\right)$ of graphs on the same vertex set $V$, a cooperative coloring is a choice of vertex sets $\left\{I_{j} \subseteq V: j \in[m]\right\}$ such that $I_{j}$ is independent in $G_{j}$ and $\bigcup_{j=1}^{m} I_{j}=V$. If all $G_{j}$ 's are the same graph $G$, then a cooperative coloring is just a proper vertex coloring of $G$ by $m$ independent sets.

A basic fact about vertex coloring is that every graph $G$ of maximum degree $d$ is $(d+1)$-colorable. It is therefore natural to ask whether $d+1$ graphs, each of maximum degree $d$, always have a cooperative coloring. This was shown to be false:

Theorem 1 (Theorem 5.1 of Aharoni, Holzman, Howard and Sprüssel [AHHS15]). For every $d \geqslant 2$, there exist $d+1$ graphs of maximum degree $d$ that do not have a cooperative coloring.

Using the fundamental result on independent transversals of Haxell [Hax01, Theorem 2], it can be shown that $2 d$ graphs of maximum degree $d$ always have a cooperative coloring. Let $m(d)$ be the minimal $m$ such that every $m$ graphs of maximum degree $d$ have a cooperative coloring. By the above, $m(1)=2$ and

$$
d+2 \leqslant m(d) \leqslant 2 d, \text { for every } d \geqslant 2 .
$$

The theorem of Loh and Sudakov [LS07, Theorem 4.1] on independent transversals in locally sparse graphs implies that $m(d)=d+o(d)$. Neither the lower bound nor the upper bound in (1) has been improved for general $d$; even $m(3)$ is not known. However, restricting the graphs to specific classes, better upper bounds can be obtained.

Definition 2. For a class $\mathcal{G}$ of graphs, denote by $m_{\mathcal{G}}(d)$ the minimal $m$ such that every $m$ graphs belonging to $\mathcal{G}$, each of maximum degree at most $d$, have a cooperative coloring.

For example, the following was proved:

Theorem 3 (Corollary 3.3 of Aharoni et al. [ABZ07] and Theorem 6.6 of Aharoni et al. [AHHS15]). Let $\mathcal{C}$ be the class of chordal graphs and let $\mathcal{P}$ be the class of paths. Then $m_{\mathcal{C}}(d)=d+1$ for all $d$, and $m_{\mathcal{P}}(2)=3$.

In this paper, we prove some bounds on $m_{\mathcal{G}}(d)$ for two more classes:

Theorem 4. Let $\mathcal{T}$ be the class of trees, and let $\mathcal{B}$ be the class of bipartite graphs. Then for $d \geqslant 2$,

$$
\begin{aligned}
\log _{2} \log _{2} d & \leqslant m_{\mathcal{T}}(d) \leqslant(1+o(1)) \log _{4 / 3} d, \\
\log _{2} d & \leqslant m_{\mathcal{B}}(d) \leqslant(1+o(1)) \frac{2 d}{\ln d} .
\end{aligned}
$$


Remark 5. Let $\mathcal{F}$ be the class of forests. It is evident that $m_{\mathcal{F}}(d) \geqslant m_{\mathcal{T}}(d)$ as $\mathcal{F} \supset \mathcal{T}$. Conversely, when $d \geqslant 2$, given $m=m_{\mathcal{T}}(d)$ forests $F_{1}, \ldots, F_{m}$ of maximum degree $d$, we can add edges to $F_{i}$ to obtain a tree $F_{i}^{\prime}$ of maximum degree $d$, and the cooperative coloring for $F_{1}^{\prime}, \ldots, F_{m}^{\prime}$ is also a cooperative coloring for $F_{1}, \ldots, F_{m}$. Therefore $m_{\mathcal{F}}(d)=m_{\mathcal{T}}(d)$ for $d \geqslant 2$.

The notions of cooperative coloring and of list coloring have a common generalization: given a system $\left(G_{1}, \ldots, G_{m}\right)$ of graphs with vertex sets $V_{1}, \ldots, V_{m}$ (which are not neccessarily the same vertex set), a cooperative list coloring is then a choice of independent sets in $G_{i}$ whose union equals $V:=V_{1} \cup \cdots \cup V_{m}$. The notion of cooperative coloring is obtained by taking $V_{i}=V$, and list colorings are formed when $G_{i}$ is an induced subgraph of the same graph $G$ for all $i$. The upper bounds in Theorem 4 generalize to cooperative list colorings. For example, our proof of Theorem 4 for bipartite graphs readily gives the following result.

Theorem 6. For every system $\left(G_{1}, \ldots, G_{m}\right)$ of bipartite graphs with maximum degree $d$ with vertex sets $V_{1}, \ldots, V_{m}$, there is a cooperative list coloring if for every $v \in V_{1} \cup \cdots \cup$ $V_{m}$, the number of its occurrences in $V_{1}, \ldots, V_{m}$, that is $\left|\left\{i \in[m]: v \in V_{i}\right\}\right|$, is at least $(1+o(1)) \frac{2 d}{\ln d}$.

A conjecture of Alon and Krivelevich [AK98, Conjecture 5.1] states that the choice number of any bipartite graph with maximum degree $d$ is at most $O(\log d)$ (see [AR08] for a result in this direction). This conjecture would follow if the term $(1+o(1)) \frac{2 d}{\ln d}$ in Theorem 6 was strengthened to $\Omega(\log d)$.

The rest of the paper is organized as follows. In Section 2 and Section 3, we prove Theorem 4 for trees and bipartite graphs respectively. In Section 4 we discuss a further generalization of cooperative colorings.

\section{Trees}

Proof of the lower bound on $m_{\mathcal{T}}(d)$. Note that the system $\mathcal{T}_{2}$, consisting of two paths in Figure 1 (one in thin red, the other in bold blue), does not have a cooperative coloring.

Suppose now that $\mathcal{S}=\left(F_{1}, F_{2}, \ldots, F_{m}\right)$ is a system of forests on a vertex set $V$, not having a cooperative coloring. We shall construct a system $Q(\mathcal{S})$ of $m+1$ new forests $F_{1}^{\prime}, F_{2}^{\prime}, \ldots, F_{m}^{\prime}, F_{m+1}^{\prime}$, again not having a cooperative coloring.

The vertex set common to the new forests is $V^{\prime}=(V \cup\{z\}) \times V$, namely the vertex set consists of $|V|+1$ copies of $V$. For every $u \in V \cup\{z\}$ and every $i \in[m]$, take a copy

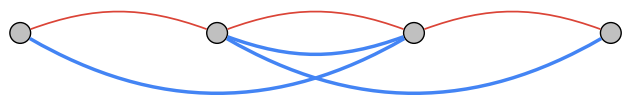

Figure 1: Construction of two paths without a cooperative coloring. 

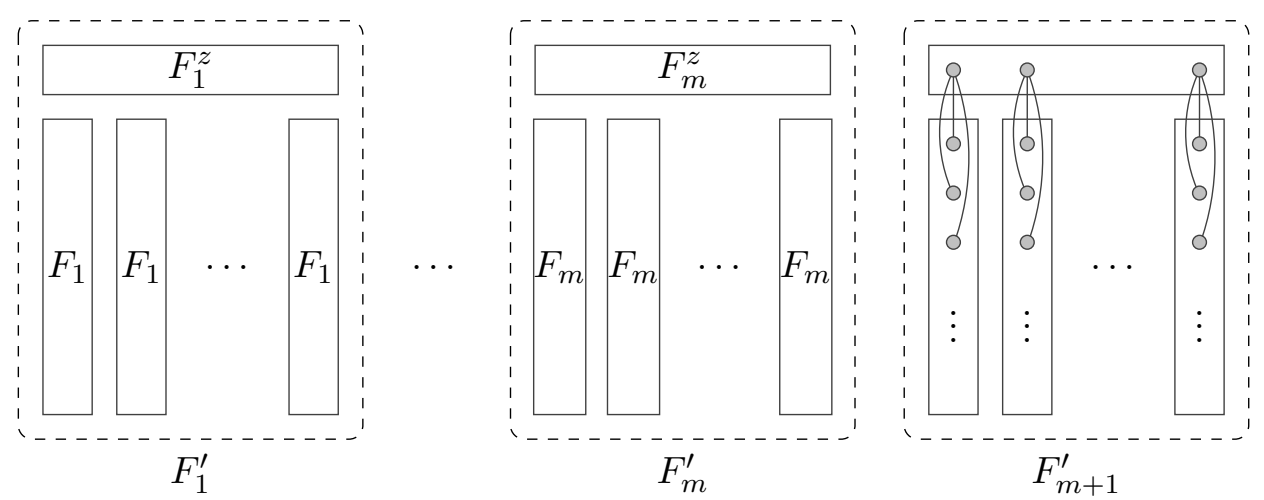

Figure 2: Construction of $Q(\mathcal{S})=\left(F_{1}^{\prime}, \ldots, F_{m}^{\prime}, F_{m+1}^{\prime}\right)$ from $\mathcal{S}=\left(F_{1}, \ldots, F_{m}\right)$.

$F_{i}^{u}$ of $F_{i}$ on the vertex set $\{(u, v): v \in V\}$. Let $F_{i}^{\prime}$ consist of $|V|+1$ disjoint copies of $F_{i}$ :

$$
F_{i}^{\prime}:=\bigcup_{u \in V \cup\{z\}} F_{i}^{u}, \quad \text { for all } i \in[m] .
$$

To these we add the $(m+1)$ st forest $F_{m+1}^{\prime}$ obtained by joining $(z, u)$ to $(u, v)$ for all $u, v \in V$. So $F_{m+1}^{\prime}$ is a disjoint union of stars, each with $|V|$ leaves.

Assume that there is a cooperative coloring $\left(I_{1}, I_{2}, \ldots, I_{m}, I_{m+1}\right)$ for the system $Q(\mathcal{S})$. Since the forests $F_{1}^{u}, F_{2}^{u}, \ldots, F_{m}^{u}$ do not have a cooperative coloring, $I_{m+1}$ must contain a vertex from $\{u\} \times V$ for all $u \in V \cup\{z\}$. In particular, $I_{m+1}$ contains a vertex $(z, u) \in I_{k+1}$ for some $u \in V$ and a vertex $(u, v)$ for some $v \in V$. Since $(z, u)$ is connected in $F_{m+1}^{\prime}$ to $(u, v)$, this is contrary to our assumption that $I_{m+1}$ is independent.

Note that $\left|V^{\prime}\right|=|V|^{2}+|V| \leqslant 2|V|^{2}$. Note also that the maximum degree of $Q(\mathcal{S})$ is attained in $F_{m+1}^{\prime}$, and it is equal to $|V|$. Recursively define the system $\mathcal{T}_{m}:=Q\left(\mathcal{T}_{m-1}\right)$ consisting of $m$ forests for $m \geqslant 3$. Because the base $\mathcal{T}_{2}$ has 4 vertices, one can check inductively that $\left|V\left(\mathcal{T}_{m}\right)\right|$ is at most $2^{3 \cdot 2^{m-2}-1}$ using $\left|V\left(\mathcal{T}_{m}\right)\right| \leqslant 2\left|V\left(\mathcal{T}_{m-1}\right)\right|^{2}$. Thus the maximum degree of $\mathcal{T}_{m}$ is at most $2^{3 \cdot 2^{m-3}-1} \leqslant 2^{2^{m-1}}$.

Given the maximum degree $d \geqslant 2$, choose $m:=\left\lceil\log _{2} \log _{2} d\right\rceil$. By the choice of $m$, the maximum degree of $\mathcal{T}_{m}$ is at most $2^{2^{m-1}} \leqslant d$. By adding a few edges between the leaves in each forest of $\mathcal{T}_{m}$, we can obtain a system of $m$ trees of maximum degree $d$ that does not have a cooperative coloring. This means $m_{\mathcal{T}}(d)>m>\log _{2} \log _{2} d$.

Proof of the upper bound on $m_{\mathcal{T}}(d)$. Let $\left(T_{1}, T_{2}, \ldots, T_{m}\right)$ be a system of trees of maximum degree $d$. We shall find a cooperative coloring by a random construction if $m \geqslant(1+$ $o(1)) \log _{4 / 3} d$.

Choose arbitrarily for each tree $T_{i}$ a root so that we can specify the parent or a sibling of a vertex that is not the root of $T_{i}$. For each $T_{i}$, choose independently a random vertex set $S_{i}$, in which each vertex is included in $S_{i}$ independently with probability $1 / 2$. Set

$$
R_{i}:=\left\{v \in S_{i} \text { : the parent of } v \text { is not in } S_{i} \text {, or } v \text { is a root }\right\} .
$$

Since among any two adjacent vertices in $T_{i}$ one is the parent of the other, $R_{i}$ is independent in $T_{i}$. 
We shall show that with positive probability the sets $R_{i}$ form a cooperative coloring. For each vertex $v$, let $B_{v}$ be the event that $v \notin \bigcup_{i=1}^{m} R_{i}$. If $v$ is the root of $T_{i}$, then $\operatorname{Pr}\left(v \in R_{i}\right)=1 / 2$; otherwise $\operatorname{Pr}\left(v \in R_{i}\right)=1 / 4$. In any case, $\operatorname{Pr}\left(v \notin R_{i}\right) \leqslant 3 / 4$, and so $\operatorname{Pr}\left(B_{v}\right) \leqslant(3 / 4)^{m}$. Notice that $B_{v}$ is only dependent on the events $B_{u}$ for $u$ that is the parent, a sibling or a child of $v$ in some $T_{i}$. Since the degree of $v$ is at most $d$, it follows that $B_{v}$ is dependent on less than $2 m d$ other events. By the symmetric version of the Lovász Local Lemma (see for example [AS16, Chapter 5]), if

$$
e \times\left(\frac{3}{4}\right)^{m} \times 2 m d \leqslant 1
$$

then with positive probability no $B_{v}$ occurs, meaning that the sets $R_{i}$ form a cooperative coloring. The inequality (2) indeed holds under the assumption that $m \geqslant(1+o(1)) \log _{4 / 3} d$.

\section{$3 \quad$ Bipartite graphs}

Proof of the lower bound on $m_{\mathcal{B}}(d)$. Given $d$, take $m=\left\lceil\log _{2} d\right\rceil$. Let the vertex set be $\{0,1\}^{m}$, and for $j \in[m]$ let $G_{j}$ be the complete bipartite graph between $V_{j}^{0}$ and $V_{j}^{1}$ where

$$
V_{j}^{k}=\left\{v \in\{0,1\}^{m}: v_{j}=k\right\}, \quad \text { for } k \in\{0,1\} .
$$

Note that the degree of $G_{j}$ is $2^{m-1} \leqslant d$.

Suppose that $I_{1}, \ldots, I_{m}$ are independent sets in $G_{1}, \ldots, G_{m}$ respectively. As each $G_{j}$ is a complete bipartite graph, $I_{j} \subseteq V_{j}^{k_{j}}$ for some $k_{j} \in\{0,1\}$. Thus $\left(1-k_{1}, \ldots, 1-k_{m}\right)$ is not in any $I_{j}$, and so $I_{1}, \ldots, I_{m}$ do not form a cooperative coloring. This means $m_{\mathcal{B}}(d)>m \geqslant \log _{2} d$.

Proof of the upper bound on $m_{\mathcal{B}}(d)$. Let $\mathcal{G}=\left(G_{1}, \ldots, G_{m}\right)$ be a system of bipartite graphs on the same vertex set $V$ with maximum degree $d$. By a semi-random construction, we shall find a cooperative coloring if $m \geqslant(1+\varepsilon) \frac{2 d}{\ln d}$ for fixed $\varepsilon>0$ and $d$ sufficiently large. We may assume that $m=O(d)$ because of $(1)$. For each $j \in[m]$, let $\left(L_{j}, R_{j}\right)$ be a bipartition of $G_{j}$. Define $J_{L}(v):=\left\{j \in[m]: v \in L_{j}\right\}$ and $J_{R}(v):=\left\{j \in[m]: v \in R_{j}\right\}$ for each vertex $v \in V$, and let $A:=\left\{v \in V:\left|J_{L}(v)\right| \geqslant m / 2\right\}$. Set $B:=V \backslash A$. Clearly, we have

$$
\begin{aligned}
& \left|J_{L}(a)\right| \geqslant m / 2, \quad \text { for all } a \in A \\
& \left|J_{R}(b)\right| \geqslant m / 2, \quad \text { for all } b \in B .
\end{aligned}
$$

Consider the following random process.

1. For each $a \in A$, choose $j=j(a) \in J_{L}(a)$ uniformly at random, and put $a$ in the set $I_{j}$.

2. For each $b \in B$, choose arbitrarily $j \in J_{R}(b) \backslash\left\{j(a): a \in A,(a, b) \in E\left(G_{j}\right)\right\}=: J_{R}^{\prime}(b)$ as long as it is possible, and put $b$ in the set $I_{j}$. 
For any $a, a^{\prime} \in A \cap I_{j}, a, a^{\prime} \in L_{j}$ and so $\left(a, a^{\prime}\right) \notin G_{j}$. This means $A \cap I_{j}$ is independent, and similarly $B \cap I_{j}$ is independent. For any $b \in B \cap I_{j}$ and $(a, b) \in E\left(G_{j}\right)$, by the definition of $J_{R}^{\prime}(b), j(a) \neq j$ and so $a \notin I_{j}$. Therefore $I_{j}$ is independent for all $j \in[\mathrm{m}]$.

To prove the existence of a cooperative coloring it suffices to show that $J_{R}^{\prime}(b)$ is nonempty for all $b \in B$ with positive probability. For a vertex $b \in B$, let $E_{b}$ be the contrary event, that is, the event that $J_{R}^{\prime}(b)$ is empty.

For a fixed $b \in B$, let us estimate from above the probability of $E_{b}$. For every $j \in J_{R}(b)$, let $E^{j}$ be the event that $j \notin J_{R}^{\prime}(b)$, that is the event that $j(a)=j$ for some $a \in A$ that is a neighbor of $b$ in $G_{j}$. For each $a \in A$ that is a neighbor of $b$ in $G_{j}$, we have

$$
\operatorname{Pr}(j(a)=j)=\frac{1}{\left|J_{L}(a)\right|} \stackrel{(3 \mathrm{a})}{\leqslant} \frac{2}{m} \leqslant \frac{\ln d}{(1+\varepsilon) d} .
$$

As there are at most $d$ neighbors of $b$ in $G_{j}$, we have for sufficiently large $d$ that

$$
1-\operatorname{Pr}\left(E^{j}\right) \geqslant\left(1-\frac{\ln d}{(1+\varepsilon) d}\right)^{d} \geqslant \exp (-(1-\varepsilon) \ln d)=d^{\varepsilon-1} \geqslant \frac{8 \ln d}{m}
$$

We claim that the events $E^{j}, j \in J_{R}(b)$, are negatively correlated. This is easier to see with the complementary events $\bar{E}^{j}, j \in J_{R}(b)$. We have to show that for any choice of indices $j_{1}, \ldots, j_{t} \in J_{R}(b)$ there holds

$$
\operatorname{Pr}\left(E^{j} \mid \bar{E}^{j_{1}} \cap \bar{E}^{j_{2}} \cap \ldots \cap \bar{E}^{j_{t}}\right) \geqslant \operatorname{Pr}\left(E^{j}\right) .
$$

The event $\bar{E}^{j_{1}} \cap \bar{E}^{j_{2}} \cap \cdots \cap \bar{E}^{j_{t}}$ means that for all $a \in A$ if $a$ is a neighbor of $b$ in $G_{j_{i}}$ then $j(a) \neq j_{i}$. Then, for any $j \notin\left\{j_{1}, \ldots, j_{t}\right\}$, for those vertices $a \in A$ that are neighbors of $b$ in $G_{j}$, knowing that $j(a) \neq j_{i}$ for certain $i \in[t]$ increases the probability that $j(a)=j$, and therefore increases the probability of $E^{j}$.

By the claim, the inequality (4) and the fact that $E_{b}=\bigcap_{j \in J_{R}(b)} E^{j}$, we have

$$
\operatorname{Pr}\left(E_{b}\right) \leqslant \prod_{j \in J_{R}(b)} \operatorname{Pr}\left(E^{j}\right) \stackrel{(3 \mathrm{~b})}{\leqslant}\left(1-\frac{8 \ln d}{m}\right)^{\frac{m}{2}} \leqslant \exp \left(-\frac{8 \ln d}{m} \cdot \frac{m}{2}\right)=\frac{1}{d^{4}} .
$$

The event $E_{b}$ is dependent on less than $m d^{2}$ other events $E_{b^{\prime}}$, since for such dependence to exist it is necessary that $b^{\prime} \in B$ is at distance at most 2 from $b$ in some graph $G_{j}$. Thus, by the Lovász Local Lemma, for the positive probability that none of $E_{b}$ occurs it suffices that

$$
e \times \frac{1}{d^{4}} \times m d^{2} \leqslant 1
$$

which indeed holds for $d$ sufficiently large as $m=O(d)$.

\section{Cooperative covers}

Cooperative coloring of graphs is a special case of a more general concept. 
Definition 7. Given a system $\left(C_{1}, \ldots, C_{n}\right)$ of (abstract) simplicial complexes, all sharing the same vertex set $V$, a cooperative cover is a choice of faces $f_{i} \in C_{i}$ such that $\bigcup_{i=1}^{n} f_{i}=V$.

A cooperative coloring for $\left(G_{1}, \ldots, G_{n}\right)$ is the special case in which $C_{i}$ is the independence complex $I\left(G_{i}\right)$ of $G_{i}$, that is, the collection of all independent sets in $G_{i}$.

Definition 8. Given a hypergraph $C$ with vertex set $V$, the edge covering number $\rho(C)$ is the minimal number of hyperedges from $C$ whose union is $V$. For a class $\mathcal{C}$ of simplicial complexes, let $n_{\mathcal{C}}(b)$ denote the minimal number $n$, such that every system $\left(C_{1}, \ldots, C_{n}\right)$ of simplicial complexes belonging to $\mathcal{C}$ on the same vertex set $V$ satisfying $\rho\left(C_{i}\right) \leqslant b$ for all $i \leqslant n$, has a cooperative cover. Let $n_{\mathcal{C}}(b)=\infty$ if no such $n$ exists.

For example, consider the class $\mathcal{I}$ of all the independence complexes of graphs. If $G$ is bipartite, then $\rho(I(G)) \leqslant 2$. Hence the fact that $m_{\mathcal{B}}(d) \geqslant \log _{2}(d)$ for all $d \geqslant 2$ (see Theorem 4) implies $n_{\mathcal{I}}(2)=\infty$.

There are natural classes $\mathcal{C}$ of hypergraphs for which $n_{\mathcal{C}}$ is finite. One of these is the class of simplicial complexes associated to polymatroids, as introduced in [Edm70]. A polymatroid $(V, r)$ is defined via a rank function $r: 2^{V} \rightarrow \mathbb{N}$, that is submodular, monotone increasing and is 0 on the empty set. A $k$-polymatroid is a polymatroid in which every singleton set has rank at most $k$. For example, a $k$-uniform hypergraph $H$ endowed with the function $r(E)=|\cup E|$, for every subset of hyperedges $E$ in $H$, is a $k$-polymatroid.

Following the notation in [LP86, Section 11], given a $k$-polymatroid $(V, r)$, a set $M \subseteq V$ is called a matching if $r(M)=k|M|$. By the submodularity of the rank function $r$, the matchings in a $k$-polymatroid form a simplicial complex on $V$, which we call the matching complex of a $k$-polymatroid.

Theorem 9. Let $\mathcal{M}_{k}$ be the class of all the matching complexes of k-polymatroids. Then $n_{\mathcal{M}_{k}}(b) \leqslant k b$ for every $b$.

The proof uses the (homotopic) connectivity $\eta(C)$ of a complex $C$. We refer to [AB06, Section 2] for background. We shall use the following two topological tools. Given a complex $C$ on $V$ and $U \subseteq V$, we denote by $C[U]$ the simplicial subcomplex induced on $U$.

Theorem 10 (Topological Hall's theorem). Let $C$ be a simplicial complex on the vertex set $V$ and let $\bigcup_{i=1}^{m} W_{i}$ be a partition of $V$. If for all $I \subseteq[m]$

$$
\eta\left(C\left[\bigcup_{i \in I} W_{i}\right]\right) \geqslant|I|,
$$

then $C$ contains a face $\sigma$ such that $\left|\sigma \cap W_{i}\right|=1$ for all $i \in[m]$.

Theorem 11. If $C$ is a matching complex on $V$ of a $k$-polymatroid, then the connectivity $\eta(C)$ of $C$ is at least $\nu(C) / k$, where $\nu(C)$ is the maximal size of faces in $C$.

The above formulation of Theorem 10 first appeared in [Mes01], attributed to the first author of the present paper (see the remark after Theorem 1.3 in [Mes01]). Theorem 11 is an unpublished result of the first two authors. The special case, where the $k$-polymatroid is the sum of $k$ matroids on the same vertex set, is proved in [AB06, Theorem 6.5]. 
Proof of Theorem 9. Let $n=k b$, and let $C_{1}, \ldots, C_{n}$ be simplicial complexes associated to $k$-polymatroids $\left(V, r_{1}\right), \ldots,\left(V, r_{n}\right)$ on the same vertex set $V$ such that the edge covering number of each $C_{i}$ is at most $b$. Let $C$ be the join of $C_{1}, \ldots, C_{n}$ on $V \times[n]$, that is,

$$
C:=\left\{\bigcup_{i=1}^{n} \sigma_{i} \times\{i\}: \sigma_{i} \in C_{i} \text { for all } i \in[n]\right\} .
$$

A cooperative cover can be viewed as a face $\sigma \in C$ such that $|\sigma \cap(\{v\} \times[n])|=1$ for all $v \in V$. By the topological Hall's theorem, it suffices to prove that

$$
\eta(C[U \times[n]]) \geqslant|U| \text { for all } U \subseteq V .
$$

Let $U$ be a subset of $V$. Note that $C_{i}[U]$ is the matching complex of the $k$-polymatroid $\left(U,\left.r_{i}\right|_{U}\right)$. By Theorem 11, $\eta\left(C_{i}[U]\right) \geqslant \nu\left(C_{i}[U]\right) / k$. Since $\nu\left(C_{i}[U]\right)$ is the maximal size of faces in $C_{i}[U]$ and the edge covering number of $C_{i}[U]$ is at most $b$, we obtain $\nu\left(C_{i}[U]\right) b \geqslant|U|$, and so $\eta\left(C_{i}[U]\right) \geqslant|U| /(k b)$. Notice that $C[U \times[n]]$ is the join of $C_{1}[U], \ldots, C_{n}[U]$. Using the superadditivity of $\eta$ with respect to the join operator and Theorem 11, we obtain the required condition for the topological Hall's theorem

$$
\eta(C[U \times[n]]) \geqslant \sum_{i=1}^{n} \eta\left(C_{i}[U]\right) \geqslant \sum_{i=1}^{n}|U| /(k b)=|U| .
$$

Remark 12. It is of interest to explore the sharpness of this result.

\section{References}

[AB06] Ron Aharoni and Eli Berger. The intersection of a matroid and a simplicial complex. Trans. Amer. Math. Soc., 358(11):4895-4917, 2006.

[ABZ07] Ron Aharoni, Eli Berger, and Ran Ziv. Independent systems of representatives in weighted graphs. Combinatorica, 27(3):253-267, 2007.

[AHHS15] Ron Aharoni, Ron Holzman, David Howard, and Philipp Sprüssel. Cooperative colorings and independent systems of representatives. Electron. J. Combin., 22(2):\#P2.27, 2015.

[AK98] Noga Alon and Michael Krivelevich. The choice number of random bipartite graphs. Ann. Comb., 2(4):291-297, 1998.

[AR08] Omid Amini and Bruce Reed. List colouring constants of triangle free graphs. In The IV Latin-American Algorithms, Graphs, and Optimization Symposium, volume 30 of Electron. Notes Discrete Math., pages 135-140. Elsevier Sci. B. V., Amsterdam, 2008.

[AS16] Noga Alon and Joel H. Spencer. The probabilistic method. Wiley Series in Discrete Mathematics and Optimization. John Wiley \& Sons, Inc., Hoboken, NJ, fourth edition, 2016. 
[Edm70] Jack Edmonds. Submodular functions, matroids, and certain polyhedra. In Combinatorial Structures and their Applications (Proc. Calgary Internat. Conf., Calgary, Alta., 1969), pages 69-87. Gordon and Breach, New York, 1970.

[Hax01] P. E. Haxell. A note on vertex list colouring. Combin. Probab. Comput., 10(4):345347, 2001.

[LP86] L. Lovász and M. D. Plummer. Matching theory, volume 121 of North-Holland Mathematics Studies. North-Holland Publishing Co., Amsterdam; North-Holland Publishing Co., Amsterdam, 1986. Annals of Discrete Mathematics, 29.

[LS07] Po-Shen Loh and Benny Sudakov. Independent transversals in locally sparse graphs. J. Combin. Theory Ser. B, 97(6):904-918, 2007. arXiv:0706.2124.

[Mes01] Roy Meshulam. The clique complex and hypergraph matching. Combinatorica, 21(1):89-94, 2001. 